\title{
Numerical Study on Heat Distribution and Transfer Characteristics of a Manifold in a Coal Mine VAM TFRR Oxidation Bed
}

\author{
Zongli Li ${ }^{1}$, Yongqi Liu ${ }^{*}, 2$, Jinhui Han ${ }^{2}$ and Zhiming Wang ${ }^{1}$ \\ ${ }^{1}$ School of Energy and Power Engineering, Shandong University, Jinan 250061, China \\ ${ }^{2}$ School of Transportation and Vehicle Engineering, Shandong University of Technology, Zibo 255049, China
}

\begin{abstract}
A thermal flow-reversal reactor is candidate for utilizing low concentration ventilation air methane. In this paper, a numerical study is performed by using the FLUENT software to explore the details of the transient preheating and starting process of the thermal flow-reversal reactor oxidation bed. The bed was heated by hot gas, which was transported and distributed through the holes of manifolds to the middle of the bed. The homogeneous porous media and coupled heat transfer models were chosen; and the mass and heat flow distributions passing through the holes, the heat transfer on the outer surface of the manifold and the temperature distribution of the bed were calculated. The results indicate that the heat of the hot gas passing through the holes decreases gradually along the direction of the hot gas flowing in the manifold, causing the temperature of the bed decrease accordingly. The calculated temperatures of the oxidation bed are compared with the tested results. The maximum error between the calculation and the test was $8.9 \%$.
\end{abstract}

Keywords: Heat flow distribution, mass flow distribution, regenerative oxidation bed, thermal reverse-flow reactor (TFRR), ventilation air methane (VAM).

\section{INTRODUCTION}

Methane vented from coal mine exhaust shafts constitutes both an unused source of energy and an atmospheric greenhouse gas $(\mathrm{GHG})$, which is 21 times more potent than Carbon Dioxide over a 100-year timeframe in trapping heat in the atmosphere [1-3]. Ventilation air exhaust streams from gassy coal mines are characterized by very large airflows, typically ranging from 47 to 470 cubic meters per second ( 100,000 to $1,000,000$ cubic feet per minute), and containing very low concentrations of methane (ranging from 0.1 to over 1.0 percent) [4]. Technologies that can reduce ventilation air methane (VAM) emissions while harnessing methane's energy offer significant benefits to the world community. The low concentration of methane in mine ventilation air is a major problem, and mitigation requires either treatment in its dilute state, or concentrating it to levels that can be used in conventional methane fuelled engines. Effective technology for increasing the concentration of methane is not available to date, but is being developed, and most work has focused on the oxidation of very low concentration methane. The thermal flow-reversal reactor (TFRR) and the catalytic flow-reversal reactor (CFRR) are both candidates for utilizing the low methane concentrations contained in VAM streams [5-8], and TFRR has been commercially used in Australia and China [9]. They employ the flow-reversal principle to transfer the heat of combustion first to a solid medium then back to incoming air in order to raise its temperature to the ignition temperature of methane.

Address correspondence to this author at the School of Transportation and Vehicle Engineering, Shandong University of Technology, Zhangzhou Road 12, Zibo 255049, China; Tel: +86-533-2782616; Fax: +86-533-2782616; E-mail: liuyq65@163.com
To start the operation, whether using the CFRR or the TFRR, the middle of the regenerative oxidation bed must be preheated to the temperature required to initiate methane oxidation or higher, and the regenerative oxidation bed must have a heat source sufficient to heat the mine ventilation air flowing in it to the auto-ignition point of methane [10]. If that temperature can be maintained in the bed, practically 100 percent conversion of methane (to carbon dioxide and water) can be achieved, the preheating and starting process can be stopped, and the reactor will run normally. A set of electric heating elements was embedded in the middle of the regenerative oxidation bed, and was used to preheating the bed by MEGTEC for the first time. It has the advantage of easy to adjust heating power. However, it sometimes was damaged by slow heat oxidation since working in the high temperature of the regenerative oxidation bed for a long time. In the past, a set of electric heating elements was embedded in the middle of the regenerative oxidation bed, and was used to initially preheat the bed by MEGTEC. This had the advantage of easily adjusting heating power. However, the elements were sometimes damaged by slow heat oxidation due to working in the high temperature of the regenerative oxidation bed for a long time.

In order to overcome this defect of the electric heating elements, a hot gas preheating system was developed by Shandong University of Technology [11]. In this system, burned gas generated from a burner, placed outside of the regenerative oxidation bed, is mixed with cool air to adjust its temperature. The hot gas is sent to the middle of the regenerative oxidation bed by a main pipe, and is distributed through many holes in the wall of manifolds arranged in the middle of the bed. In order to ensure 100 percent conversion of methane and the reaction starting completely, all the 
middle of the regenerative oxidation bed must be preheated to the auto-ignition temperature of methane. This requires the heat of the hot gas to be transported to the bed evenly as possible.

Many experimental and numerical simulation investigations on the flow distribution characteristic of manifolds have been performed, and there results indicate that the geometrical parameters of manifolds, such as inlet equivalent diameters, number and diameter of holes, have great influence on the fluid pressure distribution in the manifold, causing the change of mass flow through holes of the manifold [12-15]. However, these studies only involve the flow of liquid in manifolds without the heat transfer, and their results do not apply to transportation and distribution of the hot gas studied in this paper. During the preheating process of the regenerative oxidation bed, the flow of the hot gas is always accompanied by heat transfer. The heat transfer among the hot gas, manifold and regenerative oxidation bed is complicated, including radiation, conduction and convection, and varies with the temperature increase of the manifold and the bed. To date, the detail of the preheating of the bed using hot gas is not well understood, a basic study is necessary to clarify it.

In this study, the transient flow of the hot gas and the heat transfer among the hot gas, the manifold and the oxidation bed during the process of preheating and starting of the TFRR were simulated by using the computational fluid dynamics (CFD) program FLUENT. The porous media model and coupled heat transfer model were applied. The mass and heat flow distributions passing through the holes, the heat transfer on the outer surface of the manifold and the temperature distribution of the bed were calculated. The simulation results were verified by comparison with the measurement results.

\section{METHOD}

\subsection{Geometry}

The coal mine VAM TFRR which was made and tested at Shan Dong University of Technology was used in this study, as shown in Fig. (1). It is a simple apparatus that consists of a regenerative oxidation bed, a preheating and starting system, an upper header and a lower header, four control valves and ducts. The regenerative oxidation bed, consisting of a number of honeycomb ceramics, is the main part of the TFRR, where the methane is oxidized and the energy is stored. The honeycomb ceramic is well known for its outstanding characteristics of very low pressure drop at high mass flows, high geometrical area, and high mechanical strength. The bed is a vertical structure $(2100 \mathrm{~mm} \times 1350 \mathrm{~mm} \times$ $2400 \mathrm{~mm}$ ), in which gas flows along the vertical direction, as shown in Fig. (1). In the middle of the TFRR is the hot gas distribution system of the preheating and starting system, through which the hot gas is distributed and the oxidation bed is preheated uniformly within the starting stage. The upper header and the lower header were used to uniformly guide the feed into the oxidation bed. Four control valves were used to control the direction of VAM flowing in the regenerative oxidation bed. The operating process employs the principle of regenerative heat exchange between a gas (ventilation air) and a solid (bed of honeycomb ceramic). One cycle of the process is comprised of two flow reversals, so each flow reversal is a half-cycle. Fig. (1) assumes that during the first half-cycle both control valves 1 and 4 are open while control valves 2 and 3 are closed. Thus, the flow through the reactor takes place from the bottom to the top. After a time interval the reactor reverses the flow direction by closing the control valves 1 and 4 and opening the control valves 2 and 3 . The flow then takes place from the top to the bottom.

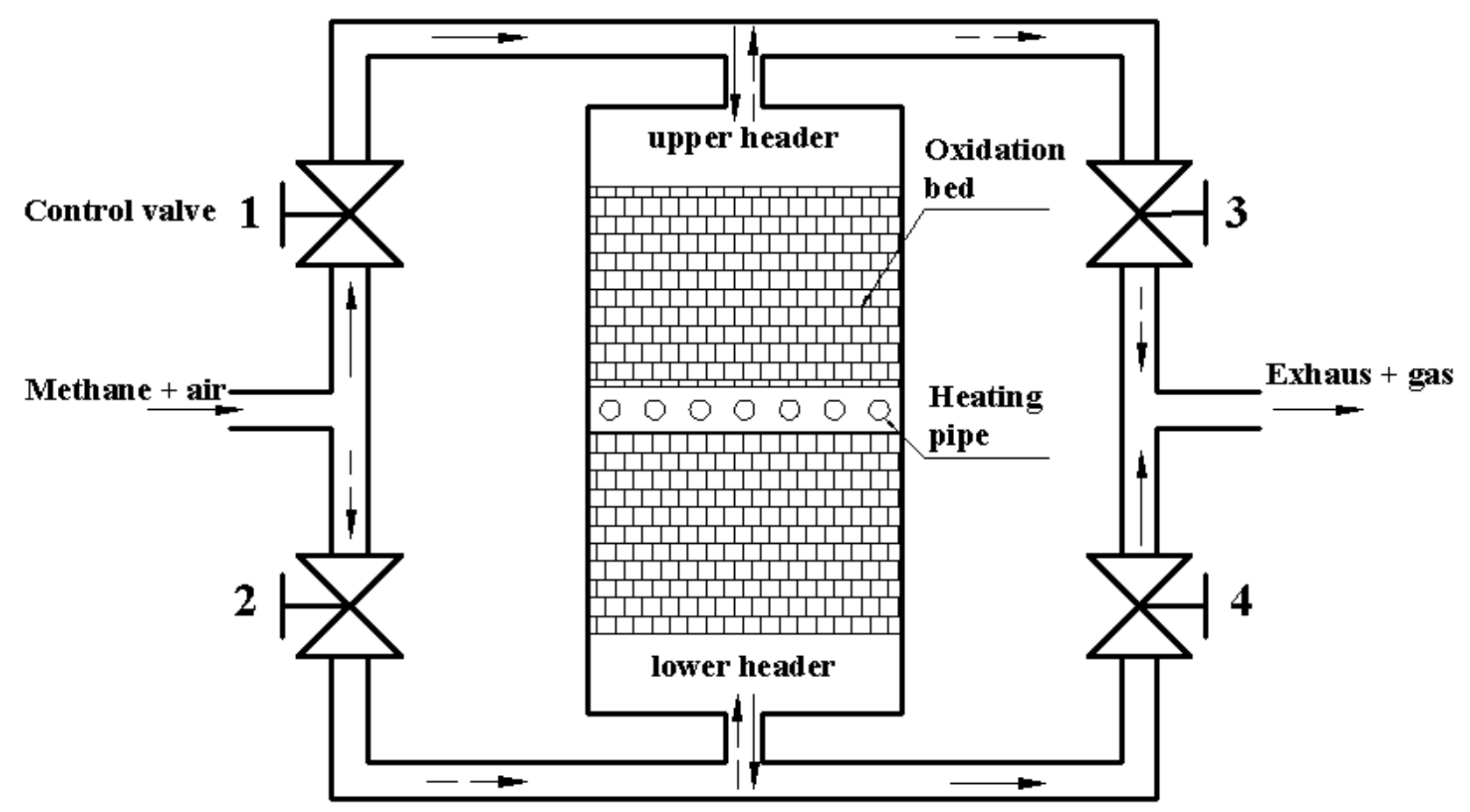

Fig. (1). The schematic of the coal mine VAM TFRR. 
However, control valves 1 and 2 are both closed during the whole preheating and starting process, and there is no ventilation air entering the regenerative oxidation bed. But control valves 3 and 4 are opened alternatively at a certain reversing interval so that the hot gas, after exchanging heat with the bed, can be emitted from the reactor, and the heat can be concentrated at the middle of the regenerative oxidation bed.

Fig. (2) shows the schematic of the preheating and starting system, which consists of two parts, one being the hot gas production and adjustment system, the other the hot gas distribution system. The burned gas coming from the burner and the air coming from the blower were first mixed in hot gas mixing chamber. In the hot gas mixing chamber, a thermocouple was used to measure the temperature of the mixing hot gas. And in order to get some certain temperature of the mixing hot gas, a valve was needed to control the mass flow of the air.

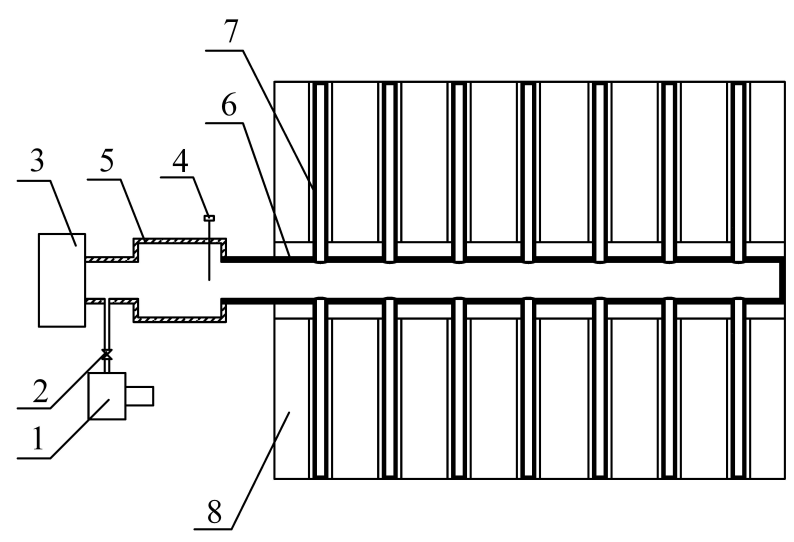

1-blower; 2-valve; 3-burner; 4-thermocouple; 5-hot gas mixing chamber; 6main pipe; 7-manifold; 8-honeycomb ceramic

Fig. (2). The schematic of the preheating and starting system.

In order to preheat the oxidation bed as uniformly as possible, many manifolds were arranged in the middle of the bed. The axis distance between two manifolds is $150 \mathrm{~mm}$. Considering the structural similarity, only one manifold and its corresponding preheating region of the oxidation bed were chosen as the simulation domain, as shown in Fig. (3). The size of the simulation domain along the oxidation bed length direction was $150 \mathrm{~mm}$. Its dimension along the oxidation bed width direction was $635 \mathrm{~mm}$ since the length of the manifold is $635 \mathrm{~mm}$. In order to further decrease the computing workload, the simulation domain height of $500 \mathrm{~mm}$ was selected from the oxidation bed center. Fig. (4) shows part of the manifold, which is made of ceramic, the same material as the bed. The inner and outer diameters are $20 \mathrm{~mm}$ and $30 \mathrm{~mm}$, respectively. There are 18 holes in the wall of the manifold, which are divided into two groups and are located at the opposite side. In other words, there are 9 holes at each side. The distance between two adjacent holes is $60 \mathrm{~mm}$. The first hole on the upper side is $70 \mathrm{~mm}$ away from the entrance of the manifold. While on the under side, it is $100 \mathrm{~mm}$ away. All the diameter of the holes is $4 \mathrm{~mm}$. The cross-sectional dimensions of the air gap between the manifold the bed are $50 \mathrm{~mm} \times 50 \mathrm{~mm}$.

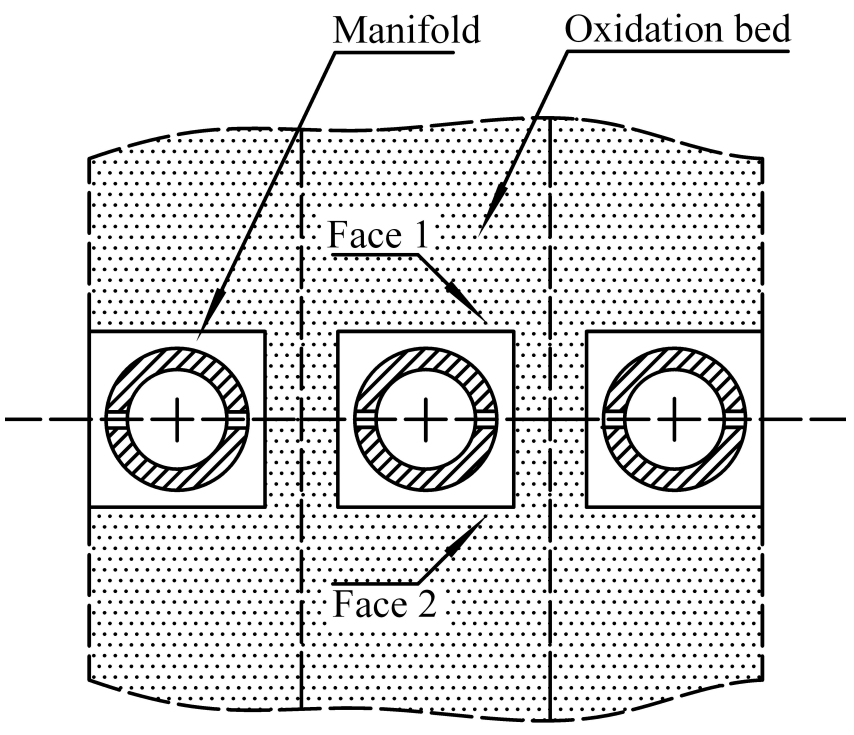

Fig. (3). The schematic of the regenerative oxidation bed.

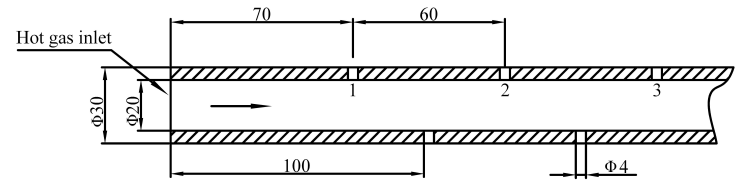

Fig. (4). The sketch of the manifold.

The heat of the hot gas is sent to the regenerative oxidation bed mainly through the two ways. The hot gas in the manifolds will flow through the holes in the wall to the outside space of the manifolds, and then into the regenerative oxidation bed, which transfers the heat directly to the bed. When the hot gas flows in the manifold, part of the hot gas heat is transferred first to the inner surface of the wall, then to the outer surface, and finally to the bed by radiation and convection heat transfer. This radiation heat transfer depends on the temperature difference between the outer surface of the manifold and the bed, and will vary during the preheating process. The hot gas heat flowing through the holes in the wall will vary accordingly. Since there is heat exchange with the wall, the temperature of the hot gas will decrease while flowing in the manifold, causing the mass and enthalpy values of the hot gas through each hole in the wall to differ.

\subsection{Numerical Modeling}

The thermophysical properties of hot gas were treated as piecewise-linear of temperature, and are listed in Table $\mathbf{1 .}$ The hot gas flow state in the manifold is different from that in the oxidation bed. The flow in the manifold and its surrounding space is turbulent since the minimum $\mathrm{Re}$ number of the hot gas is over 4000 . When the hot gas flows into the oxidation bed, the velocity of it is less than $1 \mathrm{~m} / \mathrm{s}, \mathrm{Re}$ number is small, so laminar flow happens in the oxidation bed [16]. 
Table 1. The thermophysical properties of the hot gas.

\begin{tabular}{|c|c|c|c|c|}
\hline$\frac{T}{K}$ & $\frac{\rho}{\mathrm{kg} / \mathrm{m}^{3}}$ & $\frac{c_{p}}{\mathrm{~kJ} /(\mathrm{kg} \cdot \mathrm{k})}$ & $\frac{\lambda \times 10^{2}}{W /(\mathrm{m} \cdot \mathrm{K})}$ & $\frac{\mu \times 10^{6}}{\mathrm{~kg} /(\mathrm{m} \cdot \mathrm{s})}$ \\
\hline \hline 273 & 1.293 & 1.005 & 2.44 & 17.2 \\
\hline 473 & 0.746 & 1.026 & 3.93 & 33.0 \\
\hline 673 & 0.524 & 1.068 & 6.21 & 39.1 \\
\hline 873 & 0.404 & 1.114 & 7.18 & 44.3 \\
\hline 1073 & 0.329 & 1.156 & 1.185 & 49.07 \\
\hline
\end{tabular}

For the turbulent flow, the conservation equations can be written as follows.

Mass conservation equation

$\frac{\partial \rho_{g}}{\partial t}+\frac{\partial\left(\rho u_{i}\right)}{\partial x_{i}}=0$

where $\rho_{\mathrm{g}}$ is the hot gas density, $\mathrm{kg} \cdot \mathrm{m}^{-3} ; u_{\mathrm{i}}$ is the hot gas velocity, $\mathrm{m} \cdot \mathrm{s}^{-1}$.

Momentum conservation equation

$\frac{\partial\left(\rho u_{i}\right)}{\partial t}+\operatorname{div}\left(\rho u u_{i}\right)=\operatorname{div}\left(\mu \operatorname{gradu}_{i}\right)+S_{U_{I}}-\frac{\partial P}{x_{i}}$

Energy conservation equation

$\frac{\partial(\rho h)}{\partial t}+\frac{\partial(\rho u h)}{\partial x}+\frac{\partial(\rho v h)}{\partial y}+\frac{\partial(\rho w h)}{\partial z}=\operatorname{div}(\lambda \operatorname{grad} T)+\varphi$

where $\lambda$ is the thermal conductivity of hot gas; $\phi$ is the viscous energy loss.

Here standard $k-\varepsilon$ model was used to calculate the turbulent effect. The turbulence kinetic energy $k$ and its rate of dissipation $\varepsilon$ are obtained from the following equations,

$\frac{\partial(\rho K)}{\partial t}+\rho u_{j} \frac{\partial K}{\partial x_{j}}=\frac{\partial}{\partial x_{j}}\left[\left(\mu+\frac{\mu_{t}}{\sigma_{k}}\right) \cdot \frac{\partial K}{\partial x_{j}}\right]+$

$\mu_{t} \frac{\partial u_{i}}{\partial x_{j}}\left(\frac{\partial u_{i}}{\partial x_{j}}+\frac{\partial u_{j}}{\partial x_{i}}\right)-\rho \varepsilon$

$\frac{\partial(\rho \varepsilon)}{\partial t}+\frac{\partial}{\partial x_{j}}\left(\rho u_{j} \varepsilon\right)=\frac{\partial}{\partial x_{j}}\left[\left(\mu+\frac{\mu_{t}}{\sigma_{\varepsilon}}\right) \cdot \frac{\partial \varepsilon}{\partial x_{j}}\right]+$

$\frac{c_{1} \varepsilon}{K} \mu_{t} \frac{\partial u_{i}}{\partial x_{j}}\left(\frac{\partial u_{i}}{\partial x_{j}}+\frac{\partial u_{j}}{\partial x_{i}}\right)-\frac{c_{2} \rho \varepsilon^{2}}{K}$

where $\mu_{t}=c_{\mu} \rho K^{2} / \varepsilon$. Three coefficients $\left(c_{1}, c_{2}, c_{\mu}\right)$ and three constants $\left(\sigma_{k}, \sigma_{\varepsilon}, \sigma_{T}\right)$ are listed in Table 2.

Table 2. The values of the symbols.

\begin{tabular}{|c|c|c|c|c|c|c|}
\hline Symbol & $c_{\mu}$ & $c_{1}$ & $c_{2}$ & $\sigma_{k}$ & $\sigma_{\varepsilon}$ & $\sigma_{T}$ \\
\hline Value & 0.09 & 1.44 & 1.92 & 1.0 & 1.3 & $0.9 \sim 1.0$ \\
\hline
\end{tabular}

The regenerative oxidation bed consisted of a number of honeycomb ceramics. The average equivalent diameter of each flow channel in the honeycomb ceramic is less than 3 $\mathrm{mm}$. The amount of grid required would be huge, and calculation time-consuming would be very long if each channel was accurately simulated. It can be simulated as a porous medium by using an equivalent continuum approach due to these channels being homogeneously distributed in the regenerative oxidation bed and the channel size being far smaller than the scale of the bed [17]. The thermophysical properties of the honeycomb ceramic were assumed as constants, and are listed in Table 3 . The gas flow in the bed was assumed as laminar, so the continuity equation of hot gas can be written as

$\frac{\partial \rho_{g}}{\partial t}+\nabla\left(\rho_{g} \vec{v}\right)=0$

where $v$ is the hot gas velocity, $\mathrm{m} \cdot \mathrm{s}^{-1}$.

According to the assumptions above, the momentum conservation equation of the hot gas in porous media can be described by the Darcy law

$\nabla p=-\frac{\mu}{a} \vec{v}$

in which, $\alpha$ is permeability of porous media; $\mu$ is the hot gas viscosity, $\mathrm{Pa} \cdot \mathrm{s}$

The analysis of the heat transfer in porous media requires consideration of radiation along with conduction and/or convection. There are many research results that can be used for reference [18-25]. In this study, the temperature between gas and solid is different during the preheating process, so separate energy equations for the two phases are introduced.

Solid phase energy equation is expressed as

$$
(1-\varphi)(\rho c)_{s} \frac{\partial T_{s}}{\partial t}=(1-\varphi) \nabla \cdot\left(\lambda_{s} \nabla T_{s}\right)+h\left(T_{g}-T_{s}\right)-\nabla \cdot \vec{q}
$$

in which, $T$ is temperature, $\mathrm{K} ; c$ is specific heat, $\mathrm{J} \cdot \mathrm{kg}^{-1} \cdot \mathrm{K}^{-1} ; h$ is the volumetric heat transfer coefficient, $\mathrm{W} \cdot \mathrm{m}^{-3} \cdot \mathrm{K}^{-1} ; \varphi$ is porosity; $q$ is radiation heat flux, $\mathrm{W} \cdot \mathrm{m}^{-2} ; \lambda$ is thermal conductivity of the medium, $\mathrm{W} \cdot \mathrm{m}^{-1} \cdot \mathrm{K}^{-1}$. The subscripts " $g$ " and " $s$ " stand for gas and solid phase, respectively.

The gas phase energy equation is expressed as

$\varphi\left(\rho c_{p}\right)_{g} \frac{\partial T_{g}}{\partial t}+\left(\rho c_{p}\right)_{g} V \cdot \nabla T_{g}=\varphi \nabla \cdot\left(\lambda_{g} \nabla T_{g}\right)+h\left(T_{g}+T_{s}\right)$ 
Table 3. The thermophysical properties of the honeycomb ceramic.

\begin{tabular}{|c|c|c|c|}
\hline$\frac{\rho}{k g / m^{3}}$ & $\frac{c_{p}}{k J /(k g \cdot k)}$ & $\frac{\lambda \times 10^{2}}{W /(m \cdot K)}$ & $\frac{\varphi}{\text { porosity }}$ \\
\hline \hline 2500 & 1 & 6 & 0.56 \\
\hline
\end{tabular}

The porous media in this study was considered as a gray medium. The heat source term due to radiation that appears in equation (8) is calculated by

$$
\nabla \cdot \vec{q}=\kappa\left\{4 \pi I_{b}[T(\vec{r})]-\int I(\vec{r}, \vec{s}) d \Omega\right\}
$$

where $\kappa$ is the absorption coefficient, $\mathrm{m}^{-1} ; \Omega$ is solid angle, $\mathrm{sr} ; \mathrm{I}$ is radiation intensity, $\mathrm{W} \cdot \mathrm{m}^{-2}$, and can be calculated by

$$
(\vec{s} \cdot \nabla) I(\vec{r}, \vec{s})=-\beta I(\vec{r}, \vec{s})+\kappa I_{b}(\vec{r})+\frac{\sigma_{s}}{4 \pi} \int_{4 \pi} I(\vec{r}, \vec{s}) \phi(\vec{s} \rightarrow \vec{s}) d \Omega
$$

where $\beta$ is the extinction coefficient, $\mathrm{m}^{-1} ; \sigma_{\mathrm{s}}$ is the scattering coefficient, $\mathrm{m}^{-1}$.

The heat transfer equation among the outer wall of the manifold, the hot gas and the oxidation bed is expressed as

$-\lambda\left(\frac{\partial T}{\partial n}\right)_{w}=h\left(T_{w 1}-T_{f}\right)+\varepsilon \sigma\left(T_{w 1}^{4}-T_{w 2}^{4}\right)$

where $\varepsilon$ is the emissivity of the wall surface; $\sigma$ is the Boltzmann constant, $5.67 \times 10^{-8} \mathrm{~W} /\left(\mathrm{m}^{2} \cdot \mathrm{K}^{4}\right) ; w_{1}, w_{2}$ are the outer surface of manifold and wall of oxidation bed, respectively.

\subsection{Numerical Method}

FLUENT is one of the most widely used commercial codes for simulating engineering fluid flow due to its accuracy, robustness and convenience. The threedimensional coupled heat transfer problem was solved using the software FLUENT6.2. Laminar flow was assumed for the gas flow in porous media, and the $K-\varepsilon$ turbulent model was used in the numerical calculations for the turbulent flow in the manifold. Pressure and velocity were coupled by the SIMPLE algorithm, and first order upwind discretization scheme was used. The discrete ordinate method (DOM) was used to solve the radiation transfer equation [26].

Because of great size changes for the calculation object, structured and unstructured grids were both used to discrete the simulation region. The turbulent flow domain including the manifold was meshed by an unstructured grid due to its special structural features. Part of the oxidation bed that is located between two manifold outer gaps was meshed by an unstructured grid. Other part of the oxidation bed was meshed by a structured grid. The total number of elements was 110521 .

(1) The mass flow boundary was used for the inlet boundary condition of the manifold.

For the hot air

$q_{\text {in }}=4.5 \mathrm{~kg} / \mathrm{h}, T=1273 \mathrm{~K}$

where $q_{\text {in }}, T$ are the mass flow and initial temperature of the hot gas, respectively. Initially, the porous media was at room temperature throughout.
(2) The inner surface of the manifold was coupled wall, which is defined as follows.

$T_{s}=T_{f}$ (continuous temperature)

$q_{e s}=q_{e f}$ (continuous heat flux)

The subscript $s$ and $f$ denote the solid region and fluid region, respectively.

During the preheating process, the hot gas flows into the regenerative oxidation bed through the faces 1 and 2 (see in the Fig. 3), where the turbulent flow gradually turns into the laminar flow.

$q_{e f}=q_{e b}$ (continuous mass flow)

$T_{f}=T_{b}$ (continuous temperature)

$P_{f}=P_{b}$ (continuous pressure)

The subscript $f$ and $b$ denote the fluid region and the regenerative oxidation bed region, respectively.

At the regenerative oxidation bed wall, the usual noslip and impenetrability condition was applied. These surfaces were assumed to be gray, emitting and reflecting diffusely. Therefore, the boundary intensity for outgoing direction is

$$
I(\vec{r})=\varepsilon I_{b}(\vec{r})+\frac{1-\varepsilon}{\pi} \int_{s \cdot n \leq 0} I(\vec{r}, \vec{s})|\vec{s} \cdot n| d \Omega
$$

where $n$ and $\varepsilon$ are unit vectors normal to the surface and emissivity, respectively.

(5) The top and bottom of the regenerative oxidation bed were defined as outlet, where the pressure outlet boundary is introduced due to the reverse flow of the hot gas. The operating pressure is atmospheric pressure.

Grid independence tests were performed. Starting with a total elements number of 18059 , the number of elements was increased gradually and each time the mass flows of the gas at the export of the last hole in the wall of the manifold was obtained. When the mass flows for two successive grid refinements were nearly unchanged $(0.3 \%$ difference $)$ the solutions were grid independent. The test results indicated that the total number of elements was 23875 when the solutions were grid independent.

\section{RESULTS AND DISCUSSION}

\subsection{The Distribution of Mass Flow Passing Through the Holes}

Fig. (5) shows the changes of distribution of mass flow rate passing through the holes under cold and hot conditions. 
The inlet temperatures under cold and hot conditions are 298 $\mathrm{K}$ and $1273 \mathrm{~K}$, respectively. The horizontal axis is defined as the serial number of the holes, which are counted in the direction in which the hot gas flows in the manifold, and the holes are chosen in the same side of the manifold. The vertical axis is $q_{m i} / q_{m}$, which is the ratio of mass flow rate passing through each hole and to that of the all holes (in the same side of the manifold). It can be seen from Fig. (5) that, without heating (room temperature air passing the distribution system), the distribution of mass flow is nearly uniform, and the maximum mass flow rate difference is within 0.008 . In the case of preheating, the differences of mass flow distribution among the holes are greater, and the ratio of the mass flow distribution for the holes increases along the direction in which the hot gas flows in the manifold. When the preheating time is 10 seconds, the maximum mass flow rate difference is 0.06 . But this difference decreases with the increase of preheating time. It is about 0.036 when the preheating takes 5000 seconds. The difference of the mass flow distribution of the holes is due to the variable density of the hot gas in the manifold. At the initial stage of the preheating process, the temperature of the manifold is very low; the heat transfer from the hot gas to the manifold is strong. When the hot gas flows in the manifold, the temperature gradually decreases. Thus the density of the hot gas increases accordingly, leading to the ratio of the mass flow distribution for holes increasing along the direction of the hot gas flow in the manifold. With the preheating process going on, the temperature of the manifold gradually increases; the heat transfer from the hot gas to the manifold weakens. So the temperature difference of the hot gas in the manifold decreases, resulting in a decrease in the difference of the mass flow distribution of the holes. When the temperature of the manifold is nearly equal to that of the hot gas, the temperature of the hot gas in the manifold is almost unchanged; the distribution of mass flow of holes will be stable.

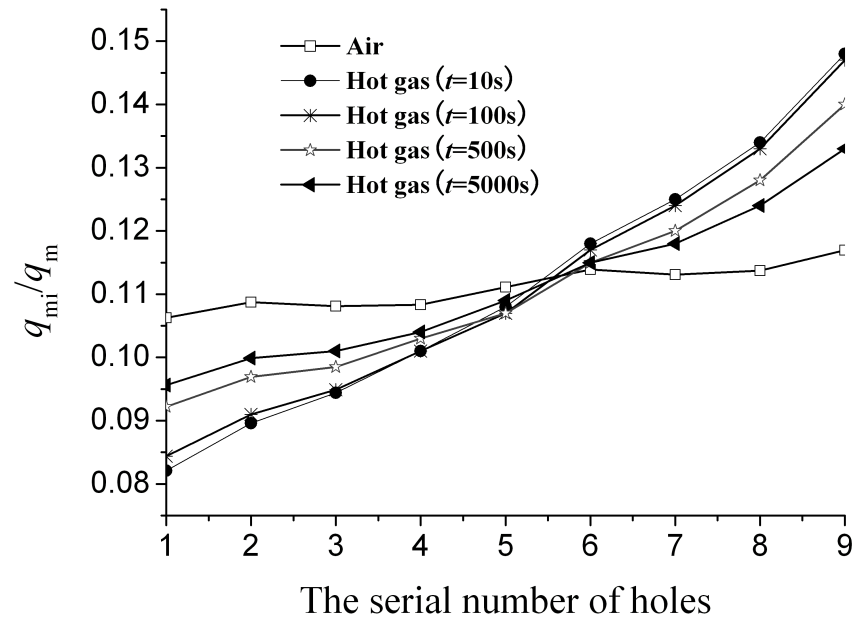

Fig. (5). The mass flow rate distribution passing through the holes.

Fig. (6) shows the difference of mass flow distribution of the holes when the preheating process is close to the stable state, that is, there is almost no temperature difference between the hot gas and the manifold. When the inlet temperature is $1273 \mathrm{~K}$, the maximum difference of $q_{m i} / q_{m}$ is 0.011 , and reduces 0.024 than that of the preheating time of 5000s by comparing Fig. (6) with Fig. (5). Also it can be seen that the difference of mass flow distribution of the holes slightly decreases with the increase of the inlet temperature, and the maximum differences of $q_{m i} / q_{m}$ are 0.013 and 0.011 under the inlet temperature of $673 \mathrm{~K}$ and $1273 \mathrm{~K}$, respectively. So the influence of the inlet temperature on the mass flow distribution is weak when the preheating process is nearly completed.

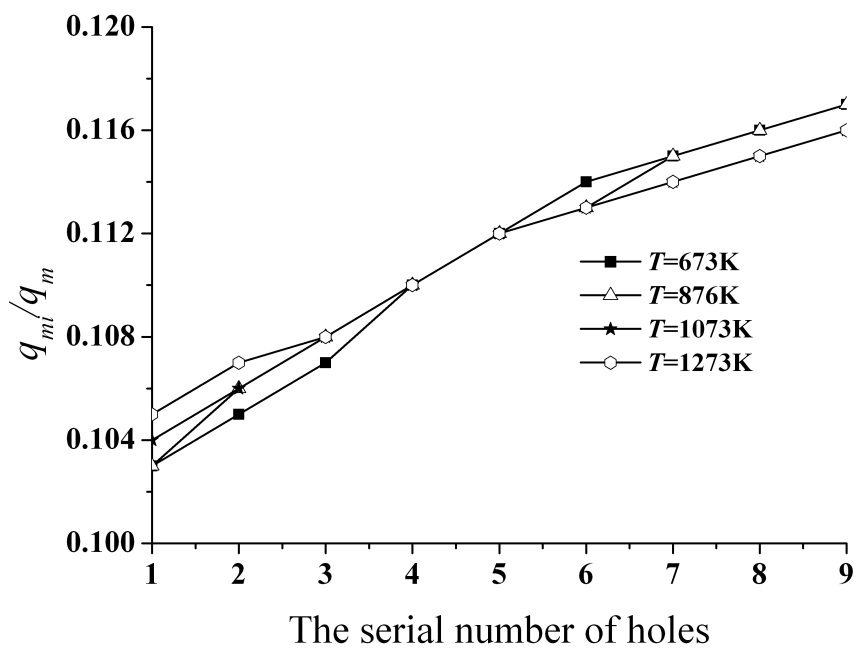

Fig. (6). The mass flow rate distribution under the condition of stable state $(\mathrm{t}=\propto)$.

\subsection{The Distribution of Heat Passing Through the Holes}

The ratio of heat rate passing through each hole and the whole is defined as $q_{e} l q_{e}$, which changes with the preheating time, as shown in Fig. (7). The first observation from Fig. (7) is that the heat passing through the first hole near the entrance of the manifold is the maximum, and the heat passing through a single hole decreases along the direction of axis of manifold (with the serial number of the holes increasing) at various preheating times. This heat distribution can be analyzed as follows. The temperature of the hot gas decreases significantly along the direction of the axis of the manifold, as shown in Fig. (8). The lower the temperature of the hot gas, the less enthalpy it owns. Although the mass flow rate passing through a single hole increases along the

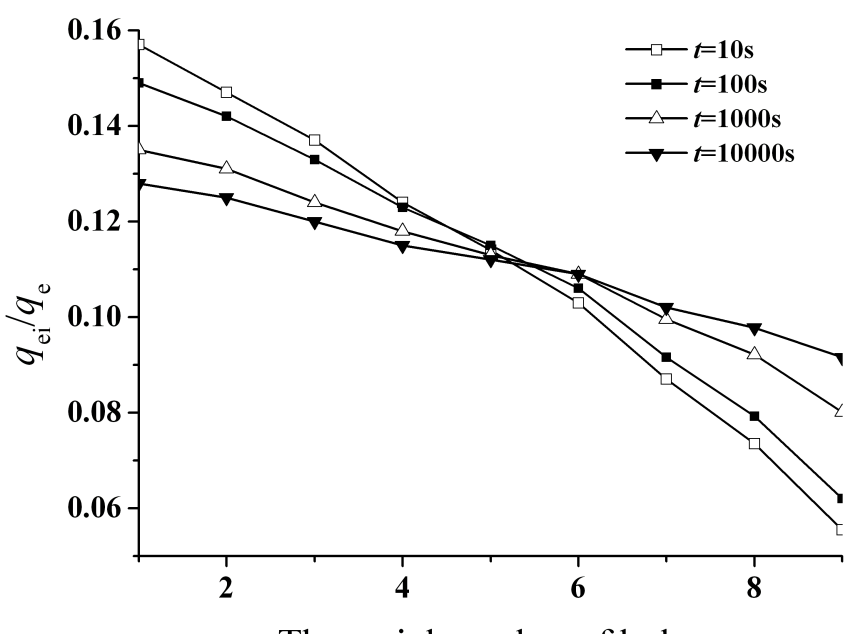

The serial number of holes

Fig. (7). The changes of $q_{e i} / q_{e}$ with the preheating time. 


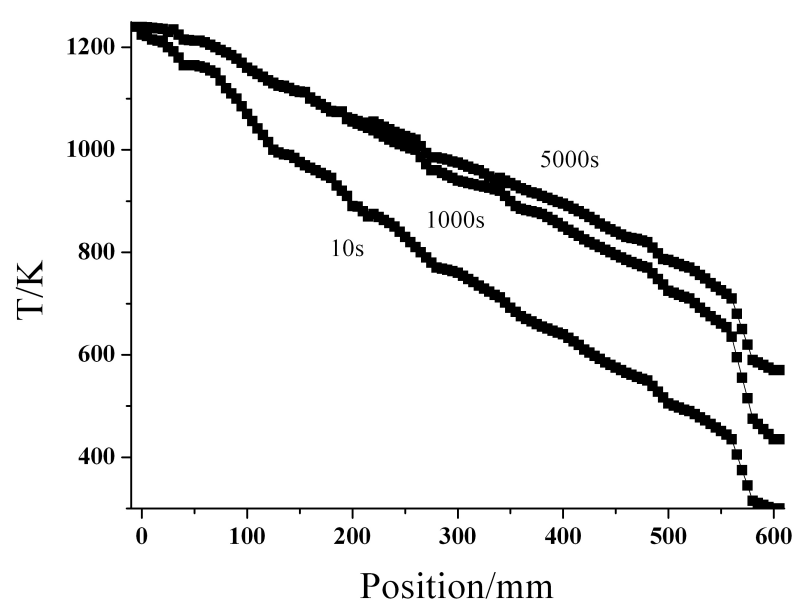

Fig. (8). The changes of temperature of the hot gas along the direction of axis of manifold.

direction of axis of the manifold, the effect of the decrease of the enthalpy is greater than that of the increase of the mass flow rate.

Another observation from Fig. (7) is that the heat passing through each hole tends to be more and more uniform as the preheating time increases. The maximum heat differences of the hot gas passing through the holes at the preheating time of $10 \mathrm{~s}, 100 \mathrm{~s}, 1000 \mathrm{~s}$ and $10000 \mathrm{~s}$ are $0.102,0.085,0.053$, and 0.036 , respectively. The reason is that the temperature difference of the hot gas in the manifold decreases with the continuing of the preheating process.

Some adjustments of the structure parameters of the manifold should be taken to increase the heat passing through the holes lying at the back-end of manifold, in order to balance the uneven heat flow distribution during the preheating process [27-29]. According to the relationship of mass flow and heat flow, if more heat passes through the holes which lie at the back-end of the manifold, more mass flow rate should transport through these. Reasonable adjustments of the structure parameters of the manifold can allow the heat to distribute uniformly; these include a gradual increase in the diameter or the number of the holes lying at the back-end of manifold to make the mass flow rate increase along the hot gas flowing direction in the manifold.

\subsection{Process of Transient Heat Transfer}

The heat of the hot gas entering in the manifold through the entrance (defined as $\Phi$ ) can be divided into two parts. One is transfered to the inner surface of the wall when the hot gas flows in the manifold; the other is transported through the holes in the wall to the outside of the manifold (defined as $\Phi_{H}$ ). Fig. (9) shows the change of ratio of the $\Phi_{H}$ and $\Phi$ with the preheating time. It can be seen that the ratio $\Phi_{H} / \Phi$ increases with the process of the preheating going on. At the initial stage of preheating, the ratio $\Phi_{H} / \Phi$ is less than $40 \%$, with most of the heat being used to preheat the manifold through convection and conduction heat transfer. But with the increases of the temperature of the manifold, the heat transfer between the hot gas and the manifold decreases, while the portion of the heat passing through the holes increases.

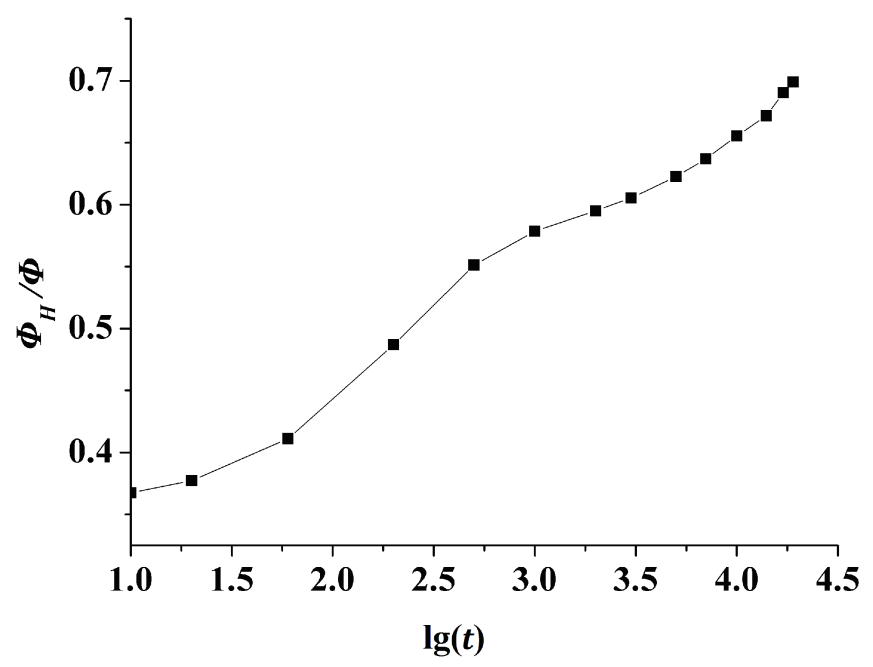

Fig. (9). The changes of $\Phi_{H} / \Phi$ with the preheating time.

The heat on the inner surface of the manifold was conducted to the outer surface of the wall. There are two heat transfer ways between the outer surface of the manifold and the bed. One way is that part of heat was transferred directly to the bed by radiation. The other is that the rest heat was transferred to the gas surrounding of the manifold by convection, then to the bed with the gas entering it. Fig. (10) illustrates the changes of the radiation heat and total heat (radiation and convection) happening on the outer surface of the manifold with increasing preheating time. In order to fully display the detail of the initial preheating stage, the abscissa (preheating time) is expressed in logarithmic form. It can be seen that the radiation heat transfer of the outer surface first increases, then decreases, and the maximum radiation heat transfer happens at about the time of 3000s. For the total heat of the outer surface, it first decreases, and quickly becomes lower than the radiation heat, then begins to increase at the preheating time of $200 \mathrm{~s}$, and reaches the

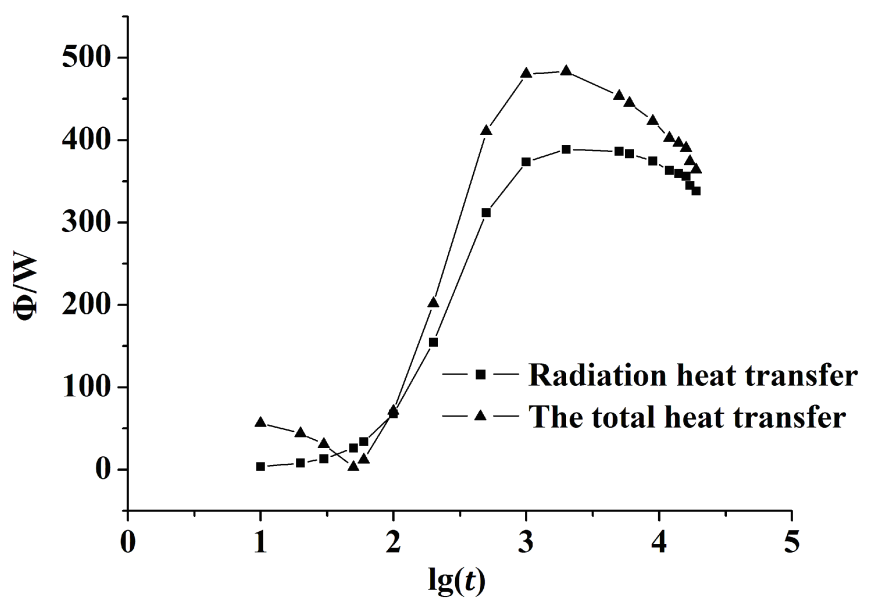

Fig. (10). The changes of the radiation heat and total heat on the outer surface of the manifold with the preheating time. 
maximum at about the preheating time of 3000 s, finally decreases again with the continuing of the preheating process. The radiation is the main form of heat transfer on the outer surface of the manifold, and accounts for more than $80 \%$ of the total heat transfer most of the time. These phenomena can be explained as follows.

The manifold and the oxidation bed are both made of high emissivity ceramic material, so the radiation heat transfer takes an important role in the preheating process. The temperature of the outer surface of the manifold is low, and the radiation heat transfer is small in the initial stage of preheating. With the process of the preheating progressing, the temperature of the outer surface of the manifold increases, while the temperature of the oxidation bed increases slowly because of the very large specific heat. So the radiation heat transfer between the manifold and the oxidation bed increases with the increases in the temperature difference between them. With the process of preheating going on, the temperature of the oxidation bed will gradually increase. At some point, the temperature difference between them reaches the maximum leading to maximum radiation heat transfer. After this, the radiation heat transfer will decrease with the decrease of the temperature difference.

The phenomenon that the total heat is lower than the radiation heat at the beginning of the preheating process indicates that the direction of the convection heat transfer between the manifold and the surrounding hot gas is from the hot gas to the manifold, which is opposite of the radiation heat transfer between the wall of the manifold and the oxidation bed. This fact means that the outer surface of the manifold absorbs heat from the surrounding hot gas, and can be explained as follows. Thermal conductivity of ceramic materials is very small, so the heat transfer in the inner of the manifold wall is slow. At the initial stage of the preheating process, the temperature of the outer surface of the manifold is lower than that of the hot gas flowing through the holes.

\subsection{Temperature Distribution of the Oxidation Bed}

The temperature distributions of the oxidation bed within the computational domain were calculated. Fig. (11) shows

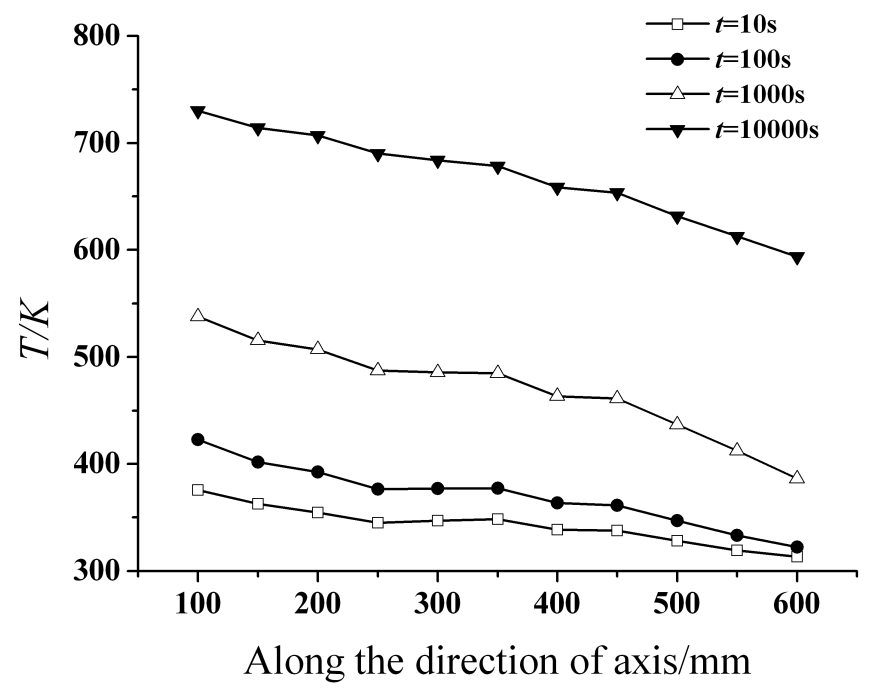

Fig. (11). The change of temperature of the oxidation bed. the temperature distributions on a line, which is $30 \mathrm{~mm}$ away from the axis of the manifold and is located on the longitudinal-section through the centerline of the manifold. It can be seen that the temperature near the center of the bed always is the maximum, and gradually decreases along the direction of the hot gas flowing in the manifold at four preheating times of $10 \mathrm{~s}, 100 \mathrm{~s}, 1000 \mathrm{~s}, 10000 \mathrm{~s}$. The maximum temperature differences of the bed at the preheating times of $10 \mathrm{~s}, 100 \mathrm{~s}, 1000 \mathrm{~s}, 10000 \mathrm{~s}$ are $47 \mathrm{~K}, 52 \mathrm{~K}, 56 \mathrm{~K}$ and $48 \mathrm{~K}$, respectively.

In order to verify the correctness of the calculation, the temperature of the oxidizer bed was tested by carrying out experiments on the coal mine VAM TFRR. Fig. (12) shows the test results. Comparing Fig. (11) with Fig. (12), the test temperatures are well consistent with the calculated results in addition to the first measuring point, which is near the center of the bed. The test temperatures of the first measuring point at the preheating times of $10 \mathrm{~s}, 100 \mathrm{~s}, 1000 \mathrm{~s}, 10000 \mathrm{~s}$ are $383 \mathrm{~K}, 429 \mathrm{~K}, 585 \mathrm{~K}$ and $786 \mathrm{~K}$, respectively, while the corresponding calculated temperatures are $369 \mathrm{~K}, 395 \mathrm{~K}$, $537 \mathrm{~K}$ and $738 \mathrm{~K}$. The maximum errors between the calculation and the test for the preheating times of $10 \mathrm{~s}, 100 \mathrm{~s}$, $1000 \mathrm{~s}, 10000 \mathrm{~s}$ are $3.8 \%, 8.6 \%, 8.9 \%$ and $6.5 \%$, respectively. The test temperatures of the first measuring point are always higher than the simulation results under the same preheating time. This is because the influence of the radiation heat transfer of the main pipe on the temperature distribution of the oxidation bed is ignored in the numerical simulation. In the actual preheating process, the temperature difference between the main pipe and the oxidation bed is large at the initial and middle preheating stage, and the radiation heat transfer between them is huge, causing the temperature of the bed near the main pipe to be higher. With the preheating process going on, the temperature of the oxidation bed is gradually increased. When the temperature of the oxidation bed closes to that of the main pipe, the main pipe has little influence on the temperature of the bed.

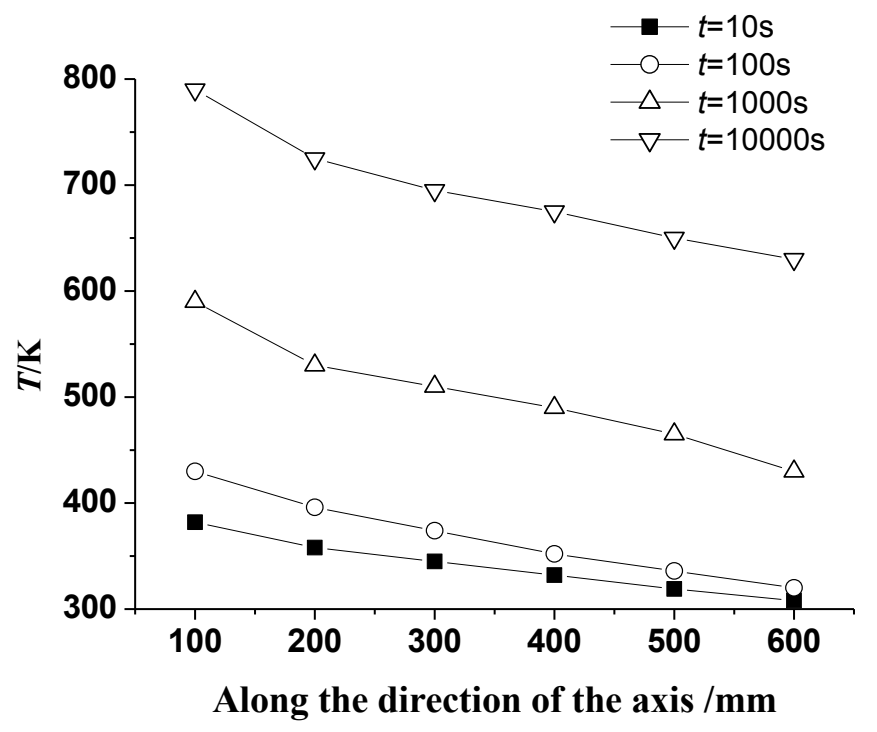

Fig. (12). The change of temperature of the oxidation bed got by experiments.

According to the above analysis, the influence of the radiation heat transfer of the main pipe on the temperature distribution of the oxidation bed should be taken into 
account in the future calculation so that the accuracy can be improved. To the further improve the design of the hot gas distribution system used in the coal mine VAM TFRR, the structure parameters of the manifold should be adjusted as follows. The distance between the first hole in the wall and the entrance of the manifold should be increased to decrease the temperature of the oxidation bed near the main pipe. The diameter or the number of the holes along the hot gas flow direction in the manifold should be gradually increased to transfer more heat to the rear oxidation bed so that the preheating uniformity of the oxidation bed can be improved.

\section{CONCLUSION}

A numerical study was performed to explore the mass flow rate and heat transfer rate distributions characteristics of a manifold and the transient heat transfer process during the preheating process of the regenerative oxidation bed used in the coal mine VAM TFRR. The main conclusions are summarized as the follows.

The mass flow of the hot gas passing through the holes in the wall increases gradually along the direction in which the hot gas flows in the manifold. While the heat flux of the hot gas passing through the holes decreases. With the preheating process going on, both the mass flow difference and the heat flux difference of the hot gas passing through the holes decrease gradually. The hot gas heat is mainly used to preheat the manifold and the heat flux passing through the holes to the oxidation bed is less than $40 \%$ of the total heat at the initial stage of the preheating process. As the temperature of the manifold increases, the heat through the holes increases. The heat transfer between the outer surface of the manifold and the oxidation bed is mainly by radiation, and reaches the maximum at the preheating time of 3000 seconds. This radiation heat transfer plays an important role in the long middle stage of the preheating process. The temperature of the oxidation bed decreases gradually along the direction of the hot gas flowing in the manifold.

In this study, only unstructured grid was used for the manifold mesh, maybe resulting in large errors of simulation results. The influence of the radiation heat transfer of the main pipe on the preheating of the oxidation bed was ignored in the numerical simulation. This will lead to calculating temperatures of the oxidizer bed near the main pipe lower than the actual values. In order to verify the correctness of the calculation, the temperatures of the oxidizer bed were tested by carrying out experiments on the coal mine VAM TFRR. The calculated temperatures of the oxidation bed are compared with the tested results. The maximum error was $8.9 \%$.

Some adjustments of the structure parameters of the manifold are suggested in future work to further improve the preheating uniformity of the oxidation bed: (1) the distance between the first hole in the wall and the entrance of the manifold should be increased; (2) the diameter or the number of the holes along the hot gas flow direction in the manifold should be gradually increased.

\section{CONFLICT OF INTEREST}

The authors confirm that this article content has no conflict of interest.

\section{ACKNOWLEDGEMENTS}

This study was financially supported by National High Technology Research and Development Program("863"Program) of China (No. 2009AA063202), Shandong Provincial Science and Technology Development Program, China (2012GGX10417) and Shandong Provincial Natural Science Foundation, China (ZR2011EL017, ZR2013EEQ005 and ZR2013EEQ008). The authors would like to express their appreciations for the above support.

\section{REFERENCES}

[1] Y.P. Cheng, L. Wang, and X.L. Zhang, "Environmental impact of coal mine methane emissions and responding strategies in China", International Journal of Greenhouse Gas Control, vol. 5, pp. 157$166,2011$.

[2] K. Warmuzinski, "Harnessing methane emissions from coal mining”, Process Safety and Environmental Protection, vol. 86, pp. 315-320, 2008.

[3] P. Marin, M.A.G. Hevia, S. Ordonez, and F.V. Diez, "Combustion of methane lean mixture reverse flow reactors: comparison between packed and structured catalyst beds", Catalysis Today, vol. 105, pp. 701-708, 2005.

[4] S. Su and J. Agnew, "Characteristics of coal mine ventilation air flows”, Journal of Environmental Management, vol. 86, pp. 44-62, 2008.

[5] K. Gosiewski, Y.S. Matros and K. Warmuzinski, "Homogeneous vs catalytic combustion of lean methane-air mixtures in reverse-flow reactors", Chemical Engineering Science, vol.63, pp. 5010-5019, 2008.

[6] C.Ö. Karacan, F.A. Ruiz, M. Cotè and S. Phipps, "Coal mine methane: a review of capture and utilization practices with benefits to mining safety and to greenhouse gas reduction", International Journal of Coal Geology, vol.86, pp. 121-156, 2011.

[7] I. Karakurt, G. Aydin and K. Aydiner, "Mine ventilation air methane as a sustainable energy source", Renewable and Sustainable Energy Reviews, vol.1, pp. 1042-1049, 2011.

[8] S. Su and J. Agnew, "Catalytic combustion of coal mine ventilation air methane", Fuel, vol. 85, pp. 1201-1210, 2006.

[9] B. Zheng, Y.Q. Liu, R.X. Liu, Z.Q. Gao and J. Meng, "Oxidation of coalmine ventilation air methane in thermal reverse-flow reactor", Journal of China Coal Society, vol. 34, pp. 1475-1478, 2009.

[10] R. Mattus, "Introduction of the VOCSIDIZER technology in power generation utilizing VAM", China Coalbed Methane, vol. 1, pp. 44-46, 2004.

[11] Y.Q. Liu, J.H. Han, Y.Y. You and Z.Q. Gao, "Numerical study on the transient preheating process of a regenerative oxidation bed", The Open Mechanical Engineering Journal, vol.5, pp. 62-67, 2011.

[12] J.Y. Wang, "Pressure drop and flow distribution in parallel-channel configurations of fuel cells: U-type arrangement", International Journal of Hydrogen Energy, vol. 33, pp. 6339-6350, 2008.

[13] J.Y. Wang, "Pressure drop and flow distribution in parallel-channel configurations of fuel cells: Z-type arrangement", International Journal of Hydrogen Energy, vol. 35, pp. 5498-5509, 2010.

[14] J.Y. Wang, "Theory of flow distribution in manifolds", Chemical Engineering Journal, vol. 168, pp. 1331-1345, 2011.

[15] S.M. Yang and Y.H. Luo, "Study of flow characteristics in manifolds", Journal of Hydrodynamics, vol.13, pp. 214-220, 1998. 
[16] H. Liu, P.W. Li and J.V. Lew, "CFD study on flow distribution uniformity in fuel distributors having multiple structural bifurcations of flow channels", International Journal of Hydrogen Energy, vol.35, pp. 9186-9198, 2010.

[17] Y. Q. Liu, Z. X. Zhang, Z. Q. Gao, R. X. Liu and B. Zheng, "Numerical simulation on resistance of the regenerative oxidation bed for ventilation air methane", Journal of China Coal Society, vol. 35, pp. 946-950, 2010.

[18] H. Amiri, S.H. Mansouri and A. Safavinejad, "Combined conductive and radiative heat transfer in an anisotropic scattering participating medium with irregular geometries", International Journal of Thermal Sciences, vol. 49, pp. 492-503, 2010.

[19] T. Prabal, S.C. Mishra and F. Durst, "Combined radiation and convection heat transfer in a porous channel bounded by isothermal parallel plates", International Journal of Heat and Mass Transfer, vol. 47, pp. 1001-1013, 2004

[20] T. Prabal, S.C. Mishra, D. Trimis and F. Durst, "Heat transfer characteristics of a porous radiant burner under the influence of a 2-D radiation field", Journal of Quantitative Spectroscopy \& Radiative Transfer, vol. 84, pp. 527-537, 2004

[21] P.X. Jiang and X.C.Lu, "Numerical simulation and theoretical analysis of thermal boundary characteristics of convection heat transfer in porous media", International Journal of Heat and Fluid Flow, vol. 28, pp. 1144-1156, 2007.

[22] K.Ichimiya and T. Matsuda, "Effects of a porous medium on local heat transfer and fluid flow in a forced convection field", Heat Mass Transfer, vol. 40, pp. 1567-1576, 1997.
[23] Z.W. Ma, V. Ramki and F. Mohammad, "Study of the gas flow distribution and heat transfer for externally manifolded fuel cell stack module using computational fluid dynamics method", Journal of Fuel Cell Science and Technology, vol. 1, pp. 49-55, 2004.

[24] X.H. Zhong, L. Zhang and C.B. Wu, "Experiments and numerical simulation of flow distribution with large flux in multi-branch pipe”, Journal of Chong Qing University, vol. 29, pp. 41-44, 2006.

[25] C.K.T. Jimmy, M.S. Ephraim and P.A. John, "Geometric strategies for attainment of identical outflows through all of the exit ports of a distribution manifold in a manifold system", Applied Thermal Engineering, vol. 29, pp. 3552-3560, 2009.

[26] M. M. Keshtkar and S. A. G. Nassab, "Theoretical analysis of porous radiant burners under 2-D radiation field using discrete ordinates method", Journal of Quantitative Spectroscopy and Radiative Transfer, vol.110, pp. 1894-1907, 2009.

[27] S.D. Chen, X.Z. Zhang and S.B. Xie, "The hydraulic design process of the perforated catch-pipe with variable aperture", Journal of Fu Zhou University, vol. 31, pp. 211-215, 2003.

[28] S. Maharudrayya, S. Jayanti and A.P. Deshpande, "Flow distribution and pressure drop in parallel-channel configurations of planar fuel cells", Journal of Power Sources, vol. 144, pp. 94-106, 2005.

[29] X.D. Wang, W.M. Yan, Y.Y. Duan, F.B. Weng, G.B. Jung and C.Y. Lee, "Numerical study on channel size effect for proton exchange membrane fuel cell with serpentine flow field", Energy Conversion and Management, vol.51, pp. 959-968, 2010.

(C) Li et al.; Licensee Bentham Open.

This is an open access article licensed under the terms of the Creative Commons Attribution Non-Commercial License (http://creativecommons.org/licenses/by-nc/3.0/) which permits unrestricted, non-commercial use, distribution and reproduction in any medium, provided the work is properly cited. 